\title{
A multidisciplinary bone metastases clinic at Toronto Sunnybrook Regional Cancer Centre - A review of the experience from 1999 to 2005
}

\author{
Kathy K Li \\ Emily Sinclair \\ Joan Pope \\ Macey Farhadian \\ Kristin Harris \\ Julie Napolskikh \\ Albert Yee \\ Lawrence Librach \\ Lesia Wynnychuk \\ Cyril Danjoux \\ Edward Chow \\ On behalf of the Bone \\ Metastases Team \\ Bone Metastases Site Group, Toronto- \\ Sunnybrook Regional Cancer Centre, \\ University of Toronto, Toronto, ON, \\ Canada
}

\begin{abstract}
Our objective in this study was to review the experience of a one-stop multidisciplinary bone metastases clinic (BMC) that offers a coordinated multidisciplinary approach to the care of cancer patients with bone metastases in a tertiary cancer centre. Patients with symptomatic bone metastases were referred to BMC and assessed by a team of specialists in various disciplines - interventional radiology, orthopedic surgery, palliative medicine, and radiation oncology. At initial consultation, patient demographics, reasons for referral, and case disposition were recorded. From January 1999 to February 2005, a total of 272 patients with bone metastases were referred to the BMC. The median age was 65 years (range 28-95) and median KPS score at consultation was 60 (range 30-90). The majority of patients came from home (74\%), while others came from a nursing home or the hospital (9\%). Almost a third (28\%) of patients had 2 or more reasons of referral, yielding a total of 354 reasons. The most common reason for referral was bone pain $(42 \%)$, bone metastases $(21 \%)$, high risk for pathological fracture $(12 \%)$, and pathological fracture $(10 \%)$. Of the 272 patients who received consultation, $40 \%$ received palliative radiotherapy, $19 \%$ received interventional surgery, $7 \%$ were referred to other support services such as palliative care, physiotherapy, and $7 \%$ had further investigation or imaging. A multidisciplinary clinic is useful for co-coordinating the management of bone metastatic disease in symptomatic patients.
\end{abstract}

Keywords: bone metastases, multidisciplinary approach, experience

\section{Introduction}

In 2006, approximately 153,100 new cases of cancer and 70,400 deaths from cancer will occur in Canada (CCS/NCIC 2006). Bone metastases are a frequent complication of cancer, and half of the individuals who died of cancer have bone metastates (Rubens 1998). Breast and prostate carcinomas are the most common to develop metastases to bone, with an incidence of $75 \%$ and 68\%, respectively (Perez et al 1990). In addition, lung, thyroid, and renal carcinoma metastasize to bone in approximately $40 \%$ of cases (Rubens 1998). With advances in effective systemic treatment and supportive care, the duration of survival of patients with bone metastases has improved substantially. Certain subsets of patients with bone metastases (eg, breast and prostate cancer with predominately bone or bone-only metastases) have life expectancies that range from two to five years. The five-year survival rate of breast cancer patients with either bone or bone-only metastases can be as high as $45 \%$, and those with bone-only metastases have a median survival of 52 months (Perez et al 1990; Yamashi et al 1991, 1992). Thus, successful management of bone metastases during these years is essential for reducing skeletal complications and for maximizing patient quality of life. The following reasons demonstrate an urgent need to reassess the management strategies in patients with bone metastases (Body 1992; Bilezikian 1992; Orr et al 1993; Chow et al 1999): 
1. Pain arising from bone metastases is the most common symptom requiring radiotherapy treatment in cancer patients;

2. The symptoms of bone metastases are often severe and develop earlier in the clinical course of patients with cancer than symptoms due to either liver or lung metastases;

3. Complications of skeletal metastases are common and can seriously impair patient quality of life and function. Pain and impaired mobility occur in $65 \%-75 \%$ of patients with bone metastases; fractures of weight-bearing long bones occur in 10\%-20\%; hypercalcemia occurs in 10\%-15\%; and spinal cord or nerve root compression occurs in 5\%;

4. Increasing incidence of bone metastases and longer survival duration of patients with bone metastases has been observed and;

5. The care of this group of patients has been poorly integrated and coordinated.

Fifty to $75 \%$ of patients with bone metastases suffer from severe pain (Cleeland et al 1996). As reflected in a consensus statement from a National Cancer Institute workshop, the under-treatment of cancer pain is a serious and often neglected public health problem (Jacox et al 1994). Treatment for symptomatic bone metastases often involves multiple care providers including medical and radiation oncologists, pain specialists, surgeons, and other health care professionals. Clinical trials in various disciplines addressing the optimal management of bone metastases are under way and treatment options for bone metastases have been expanding as reflected in the latest clinical trials testing the newer generations of bisphosphonates (Weinfurt et al 2006) and increased use of orthopedic surgery.

Radiotherapy and orthopedic stabilization are effective palliative measures for bone metastases. Singer commented that "bone pain from mechanical effects often necessitates long term treatment with strong analgesics" (Singer 1997). In response, Krikler (1997) stated that the most effective solution for mechanical pain is surgery. Galasko (1997) reported that surgical stabilization of such lesions often gives the patient total pain relief and may obviate the need for analgesics. Although such therapies are effective in alleviating pain, specialists who deliver the treatments often work independently, leading to the lack of a coordinated treatment. This is evident in the review by O'Donoghue and colleagues (1997) of 269 women with breast cancer who had developed bone metastases. Eighty- two episodes of structurally significant bone destruction in 47 women were identified. The researchers concluded that clinical review by an orthopaedic surgeon would have been appropriate in $89 \%$ of the episodes, but such a review was sought in only $46 \%$. Surgery would have been feasible for $65 \%$ of the episodes, but was carried out in only $31 \%$. Bracing would have been appropriate in $40 \%$ of episodes, but was provided in only $18 \%$.

To date, only one published report exists that describes an integrated clinic model for the multidisciplinary management of bone metastases. The clinic was developed at the University of Texas M.D. Anderson Cancer Centre to provide an effective and comprehensive management of bony metastases (McQuay et al 1999). In total, presenting symptoms and the extent of disease of 108 patients were retrospectively evaluated. Presenting symptoms were evaluated with the Wisconsin Brief Pain Inventory. Patients were assessed by staff physicians from diagnostic radiology, nuclear medicine, pain and symptom management, physical medicine and rehabilitation, orthopedic surgery, medical oncology and radiotherapy. The assessment included a review of past therapies, evaluation of current treatment options and consideration of underlying medical conditions. Janjan and colleagues (1998) suggested that the use of multidisciplinary coordination of care can help to overcome many practical difficulties and address specific problems in the management of metastatic disease in symptomatic patients.

The Division of Orthopedics at Sunnybrook Health Sciences Centre and the Rapid Response Radiotherapy Program at Toronto Sunnybrook Regional Cancer Centre initiated a first-of-its-kind clinic, the Bone Metastases Clinic, at the Toronto-Sunnybrook Regional Cancer Centre in January 1999. The clinic aims to provide a coordinated multidisciplinary approach to the care of cancer patients with bone metastases. This multidisciplinary service will also save the time and effort that a patient would otherwise expend during separate, sequential visits to various specialists for consultation.

This paper is to report our experience at the Bone Metastases Clinic since its inception in 1999.

\section{Methods}

The BMC is managed by a team of specialists in various disciplines: interventional radiology, nursing, orthopedic surgery, pain and palliative medicine, radiation oncology, and radiation therapy. The clinic sees patients every second and fourth Friday of each month.

A prospective database has been set up for patients referred for joint consultation at BMC since January 1999. The referral required a pathological diagnosis of cancer and documentation of bone metastatic disease either by 
pathological confirmation, clinical examination or imaging studies. Patients were enrolled into the database if they were able to speak English, give verbal consent and respond to questions that assessed their symptoms. Patients were excluded from the database if they were confused, refused, or were unable to complete the symptom assessment.

During the initial consultation, patient demographics, cancer history, disease status and symptom profiles were collected. Patients were asked to rate their symptom distress using the Edmonton Symptom Assessment Scale (ESAS) with an 11 point categorical scale (0-10, $0=$ lack of symptom, $10=$ worst possible symptom). The scale evaluates nine symptoms (pain, fatigue, nausea, depression, anxiety, drowsiness, appetite, sense of well-being, and shortness of breath). Analgesic consumption, site(s) of bone metastases and the risk of fractures at the first visit were assessed and recorded. The functional status of the patient was scored as follows: normal with pain free use of the extremity and spine; normal use with pain; significant limited use (eg, use of prosthesis, walker, cane, crutches, and sling); and nonfunctional extremity/ spine (wheelchair bound, bedridden).

Patients were assessed by an orthopaedic surgeon, a radiation oncologist and a pain specialist at the consultation. The team then made a joint recommendation based on their assessments. Nursing and radiation therapy support was also provided. If the patient was a candidate for percutaneous vertebroplasty, an interventional radiologist was consulted.

\section{Results}

\section{Patient characteristics and baseline symptom distress}

From January 1999 to February 2005, a total of 272 patients with bone metastases were referred to the BMC. One hundred and seven patients were female $(54 \%)$ and 125 were male (46\%). Their median age was 65 years and the three most common primary sites were breast (31\%), lung (21\%) and prostate (11\%). The median KPS score at consultation was 60 (range 30-90). The majority of patients (74\%) arrived from their home, and only $9 \%$ arrived from a hospital or a hospice facility. About $10 \%$ of the patients had significant weight loss, which was defined as $\geq 10 \%$ over the last 6 months (Table 1). A total of 354 reasons for referral were recorded, where almost a third $(28 \%)$ of patients had 2 or more reasons of referral. The top three reasons for referral were bone pain (42\%), bone metastases (21\%) and high risk
Table I Patient characteristics $(n=272)$

\begin{tabular}{|c|c|}
\hline \multicolumn{2}{|l|}{ Sex } \\
\hline Male & I 47 (54\%) \\
\hline Female & $125(46 \%)$ \\
\hline \multicolumn{2}{|l|}{ Age (years) } \\
\hline Median (range) & $65(28-95)$ \\
\hline \multicolumn{2}{|l|}{ Primary cancer site } \\
\hline Breast & $84(31 \%)$ \\
\hline Lung & $56(21 \%)$ \\
\hline Prostate & 30 (II\%) \\
\hline Multiple Myeloma & $21(8 \%)$ \\
\hline Kidney & $20(7 \%)$ \\
\hline Unknown & $21(7 \%)$ \\
\hline Gastrointestinal & $16(6 \%)$ \\
\hline Others & 24 (9\%) \\
\hline $\begin{array}{l}\text { Weight loss }>10 \% \text { over the last } \\
6 \text { months }\end{array}$ & $22(10 \%)$ \\
\hline \multicolumn{2}{|l|}{ Karnofsky performance score } \\
\hline Median (range) & $60(30-90)$ \\
\hline \multicolumn{2}{|l|}{ Painful bony sites } \\
\hline Spine & III (4I\%) \\
\hline Pelvis/Hips & $70(26 \%)$ \\
\hline Lower limbs & 45 (I7\%) \\
\hline Upper limbs & $35(13 \%)$ \\
\hline Trunk & $8(3 \%)$ \\
\hline Others & $3(1 \%)$ \\
\hline \multicolumn{2}{|l|}{ Patient arrived from } \\
\hline Home & $200(74 \%)$ \\
\hline Hospital/Hospice & $24(9 \%)$ \\
\hline Other & $9(3 \%)$ \\
\hline Unknown & $39(14 \%)$ \\
\hline
\end{tabular}

for pathological fracture (12\%). Patients identified the spine (41\%), pelvis and hips (26\%), lower limbs (17\%), and upper limbs (13\%) as the most painful bony sites (Table 2).

Table 3 summarizes the ESAS scores at initial consultation. Fatigue and the sense of wellbeing were the two most severe

Table 2 Reason(s) for referral*

\begin{tabular}{ll}
\hline Bone pain & $147(42 \%)$ \\
Bone metastases & $76(21 \%)$ \\
High risk for pathological fracture & $41(12 \%)$ \\
Pathological fracture & $34(10 \%)$ \\
Spinal cord compression & $20(5 \%)$ \\
Other pain & $16(5 \%)$ \\
Others & $20(6 \%)$ \\
\hline
\end{tabular}

Note: $* 354$ reasons in total for $n=272$. 
Table 3 Symptom distress according to the Edmonton symptom assessment scale

\begin{tabular}{|c|c|c|c|c|c|}
\hline Symptom & $\mathbf{N}$ & Mean & Standard deviation & Median & Range \\
\hline Pain & 100 & 3.88 & 3.31 & 3.0 & $0-10$ \\
\hline Fatigue & 90 & 4.50 & 2.72 & 4.0 & $0-10$ \\
\hline Nausea & 91 & 1.09 & 1.88 & 0.0 & $0-9$ \\
\hline Depression & 83 & 2.75 & 2.57 & 3.0 & $0-10$ \\
\hline Anxiety & 87 & 3.33 & 2.77 & 3.0 & $0-10$ \\
\hline Drowsiness & 88 & 2.50 & 2.60 & 2.0 & $0-10$ \\
\hline Appetite & 90 & 3.28 & 3.26 & 2.5 & $0-10$ \\
\hline Sense of well-being & 86 & 3.95 & 2.59 & 3.5 & $0-10$ \\
\hline Shortness of breath & 90 & 1.86 & 2.36 & 1.0 & 0-9 \\
\hline
\end{tabular}

Notes: 0 , lack of symptom; 10, worst possible symptom.

symptoms that patients experienced (median of 4 and 3.5, respectively). Pain, depression and anxiety were also bothersome, with a median score of 3 .

\section{Orthopaedic assessment}

At consultation, the staff orthopaedic surgeon's assessment was recorded. The functional status of the affected extremity/spine, the presence or risk of fractures and the severity of the risk was recorded. The report was completed only about $50 \%$ of the time $(n=137)$. The records show that $35 \%$ of the patients reported significant limited use of their affected extremity or spine (as evident in the use of prosthesis, walker, cane, crutches and sling), 25\% reported normal use with pain, $15 \%$ reported complete nonfunctional use (wheelchair-bound or bedridden) and 14\% had normal and pain free use (Table 4).

When assessed for the presence or risk of fractures, it was found that $47 \%$ of patients were at a high risk of fractures, $34 \%$ were at low risk and $12 \%$ presented with a fracture at consultation (Table 5). Of the 65 patients in high risk, $34(52 \%)$ of them had a high risk of fractures at the extremities, where 22 of them (65\%) presented with a lytic lesion that was $>2.5 \mathrm{~cm}$ in size at their extremities. Thirty patients (46\%) were classified to have a high risk of fracture at the spine because of mechanical instability (16/30), spinal cord

Table 4 Functional status of extremity/spine $(n=137)$

\begin{tabular}{ll}
\hline Status & \\
\hline Significant limited use & $48(35 \%)$ \\
Normal use with pain & $34(25 \%)$ \\
Nonfunctional & $20(15 \%)$ \\
Normal, pain-free use & $19(14 \%)$ \\
Unknown & $16(12 \%)$ \\
\hline
\end{tabular}

compression (12/30), cauda equina syndrome or more than two nerve root deficits $(2 / 30)$.

\section{Case dispositions and recommendations}

After thorough examination and evaluation of a patient's symptoms, the BMC team discussed to suggest the best course of action. Of the 272 patients who received consultation, $40 \%$ received palliative radiotherapy, $19 \%$ received interventional surgery, $7 \%$ were referred to other support services such as physiotherapy, and $7 \%$ had further investigation or imaging completed (Table 6).

\section{Discussions}

Optimal management of structurally significant bony metastases often requires an overall effort to restore and maintain function, as well as relieve cancer-related pain. Pain is the most frequent symptom of bone metastases. It develops slowly and becomes progressively more severe. In the early stages, pain can mostly be controlled with acetaminophen, anti-inflammatory agents and opiates. Despite being on a stable opioid treatment regimen, incident pain (a transitory flare of pain upon movement) is a common compliant among patients with bone metastases (Portenoy and Hagen 1990). This type of pain restricts patients from normal activities and interferes with their ability to function.

When pain becomes progressively worst and patients fail to respond to drug therapy, alternative therapies must be considered. For the majority of patients with bone metastases, radiotherapy provides excellent palliation for localized bone pain. In $66 \%$ of patients, radiotherapy provided partial pain relief after a median time of 3 to 4 weeks (McQuay et al 1999). However, patients may also experience episodes of an intensification of pain (pain flare) shortly after radiotherapy. About $14 \%$ of the patients receiving single dose 
Table 5 Presence of bone metastasis fractures/risk of fracture ( $\mathrm{n}=137)$

\begin{tabular}{ll}
\hline Presence of fracture & \\
Pathological & $14(10 \%)$ \\
Wedge & $3(2 \%)$ \\
Total presence of fracture: & $17(12 \%)$ \\
Low risk & $46(34 \%)$ \\
High risk & \\
Extremities: $50 \%-74 \%$ cortex involved & $5(4 \%)$ \\
$\geq 75 \%$ cortex involved & $6(4 \%)$ \\
Lytic lesion 2.5 to 5 cm in size & $11(8 \%)$ \\
Lytic lesion $\geq 5$ cm in size & $5(4 \%)$ \\
Subtrochanteric region of femur & $7(5 \%)$ \\
Spine: Mechanical instability & $16(12 \%)$ \\
Cauda equine syndrome OR $\geq 2$ & $2(1 \%)$ \\
nerve root deficits & $12(9 \%)$ \\
Spinal cord compression & $1(1 \%)$ \\
No reason: & $65(47 \%)$ \\
Total high risk & $9(7 \%)$ \\
None &
\end{tabular}

of radiation experience pain flare on both day one and day two (Chow et al 2005). Thus, modifications to the patient's analgesic regimen are necessary to control symptoms both during and after radiotherapy. However, the pain relieving effect of radiotherapy is not permanent. A median time to pain progression is 5 to 6 months following radiotherapy (Steenladn et al 1999), and about $40 \%$ of patients experience an increase in pain after one year (BPT 1999). Retreatment is often also required when the effects of radiotherapy wears off. In a large RCT (Steenland et al 1999), 16\% of all patients required re-treatment. The rate of retreatment was significantly more pronounced in patients who previously received a single fraction than those who received multiple fractions regimens $(25 \%$ vs $7 \%)$. Other than palliating pain, radiotherapy can also be used to prevent a pathologic fracture and treat neurological complications from spinal cord compression (Blum et al 2003). However, when radiotherapy

Table 6 Case disposition and treatment recommendations $(\mathrm{n}=280 *)$

\begin{tabular}{ll}
\hline Radiotherapy & $108(40 \%)$ \\
Surgery & $53(19 \%)$ \\
Other support services & $19(7 \%)$ \\
Further investigation and imaging & $18(7 \%)$ \\
No action & $69(25 \%)$ \\
Others & $13(4 \%)$ \\
\hline
\end{tabular}

Notes: $* 4$ patients received two treatment recommendations. is inadequate to palliate pain or manage such complications, surgery may be indicated.

"Orthopedic surgery is often prescribed to palliate bone pain and restore function. Patients can benefit from procedures such as intramedullary nailing, or prophylactic internal fixation when the risk of pathological fracture is significant. To prevent further tumor growth and bone loss, radiotherapy should follow surgery. Newer minimally invasive procedures such as percutaneous vertebroplasty and kyphoplasty are also highly effective in treating painful vertebral compressive fractures and preventing future vertebral collapse."

-(Forney et al 2003)

The complicated disease trajectory in patients with bone metastases necessitates a multidisciplinary effort between various specialties to provide symptom relief, maintain or restore function, and improve patients' quality of life. Effective and comprehensive management approaches are imperative to ensure patients receive effective symptom and pain control and are assessed for: i) the risk of other complications that require emergent interventions and ii) the need for other therapies and support service. The Toronto Sunnybrook Regional Cancer Centre Bone Metastases Clinic demonstrates that the integration of services allows for more streamlined care and eliminates the inconvenience of separate visits to various specialists for consultation. With one visit, the patient benefits from a multidisciplinary evaluation that assesses the appropriate intervention to palliate the presenting symptoms (Figure 1). Patients can also benefit from an additional resource: a 40-page educational binder titled "Helping You to Help Yourself"

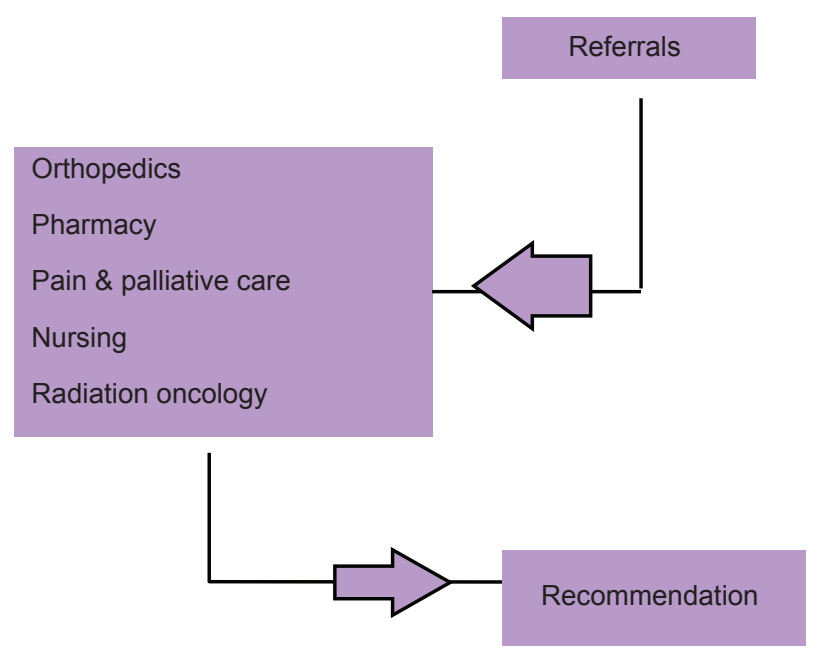

Figure I Bone metastases clinic. 
Pope 2004). The resource book was developed specifically for patients with bone metastases by the BMC team and the nurse coordinator of the clinic. Contents include treatment information, coping strategies, tips on effective management of pain and fatigue, community resources, bone metastases diagnosis information and relevant case studies (Pope 2004).

One limitation of the study is the incomplete data on symptom distress measured by the ESAS and recorded on orthopaedic assessments. Modifications to patients' analgesic regimen should also be carefully recorded. Future studies should investigate improved methods of collecting the information, examining quality of life measures, patient satisfaction and the cost-effectiveness of the clinic.

\section{Acknowledgments}

We thank Mrs Melissa Frost for the secretarial assistance. The study was supported by the Michael and Karen Goldstein Cancer Research Fund.

\section{References}

Bilezikian J. 1992. Management of acute hypercalcemia. N Engl J Med, 326:1196-203.

Blum RH, Novetsky D, Shasha D, et al. 2003. The multidisciplinary approach to bone metastases. Oncology (Williston Park), 17:845-7.

Body J. 1992. Metastatic bone disease: Clinical and therapeutic aspects. Bone, 13(Suppl 1):S57-62.

[BPT] The Bone Pain Trial Working Party. 1999. 8 Gy single fraction radiotherapy for the treatment of metastatic skeletal pain: randomized comparison with a multifraction schedule over 12 months of patient follow-up. Radiother Oncol, 52:111-23.2.

[CCS/NCIC] Canadian Cancer Society/National Cancer Institute of Canada. 2006. Canada Cancer Statistics 2006. Toronto, Canada: Canadian Cancer Society/National Cancer Institute of Canada.

Chow E, Finkelstein J, Connolly R, et al. 1999. New combined bone metastases clinic: the ultimate one-stop for cancer patients with bone metastases. Curr Oncol, 7:205-7.

Chow E, Ling A, Davis L, et al. 2005. Pain flare following external beam radiotherapy and meaningful change in pain scores in the treatment of bone metastases. Radiother Oncol, 75:64-9.

Cleeland C, Gonin R, Hatfield A, et al. 1996. Pain and its treatment in outpatients with metastatic cancer. $N$ Engl J Med, 330:592-6.
Fourney DR, Schomer DF, Nader R, et al. 2003. Percutaneous vertebroplasty and kyphoplasty for painful vertebral body fractures in cancer patients. J Neurosurg, 98:21-30.

Galasko C. 1997. Multiple myeloma: surgical stabilisation often provides good pain relief. $B M J, 315: 187$

Jacox A, Carr D, Payne R, et al. 1994. Management of cancer pain, clinical practice guideline No. 9. AHCPR Publication No. 94-0592. Rockville, MD. Agency for Health Care Policy and Research, US Department of Health and Human Services, Public Health Service.

Janjan N, Payne R, Gillis T, et al. 1998. Presenting symptoms in patients referred to a multidisciplinary clinic for bone metastases. $J$ Pain Symptom Manage, 16:171-8.

Krikler S. 1997. Multiple myeloma: surgery is often more effective than analgesia for mechanical pain. BMJ, 315:186.

McQuay HJ, Collins SL, Carroll D, et al. 1999. Radiotherapy for the palliation of painful bone metastases. Cochrane Database Syst Rev, 3:CD001793.

O’Donoghue D, Howell A, Bundred N, et al. 1997. Orthopedic management of structurally significant bone destruction in breast cancer bone metastases. J Bone Joint Surg, 79B(suppl 1):98.

Orr F, Kostenuik P, Sanchez-Sweatman O, et al. 1993. Mechanisms involved in the metastasis of cancer to the bone. Breast Cancer Res Treat, 25:151-63.

Perez J, Machiavelli M, Leone B, et al. 1990. Bone-only versus visceral-only metastastic pattern in breast cancer: analysis of 150 patients. Am J Clin Oncol, 13:294-8

Pope J. 2004. Helping you to help yourself: an educational binder to help you and your family understand, live and cope with bone metastases. Toronto: Toronto Sunnybrook Regional Cancer Centre.

Portenoy RK, Hagen NA. 1990. Breakthrough pain: definition, prevalence and characteristics. Pain, 41:273-81.

Rubens R. 1998. Bone metastases - the clinical problem. Eur J Cancer, 34:210-3.

Singer C. 1997. ABC of clinical haematology: multiple myeloma and related conditions. $B M J, 314: 960-3$.

Steenland E, Leer JW, van Houwelingen H, et al. 1999. The effect of a single fraction compared to multiple fractions on painful bone metastases: a global analysis of the Dutch Bone Metastasis Study. Radiother Oncol, $52: 101-9$

Weinfurt K, Anstrom K, Castel L, et al. 2006. Effect of zoledronic acid on pain associated with bone metastasis in patients with prostate cancer. Ann Oncol, 17:986-9.

Yamashia K, Ueda T, Komatsubara Y, et al. 1991. Breast cancer with boneonly metastases: visceral metastases - free rate in relation to anatomic distribution of bone metastases. Cancer, 68:634-7.

Yamashia K, Ueda T, Komatsubara Y, et al. 1992. A classification of bone metastases from breast cancer. In: Uchida A, Ono K (eds). Recent advances in musculoskeletal oncology. Berlin: Axel Springer Verlag AG, p. 254. 